\title{
Inducible defenses and rotifer food chain dynamics
}

\author{
Irene Van der Stap · Matthijs Vos - Wolf M. Mooij
}

(C) Springer Science+Business Media B.V. 2007

\begin{abstract}
Theoretical studies have predicted that inducible defenses affect food chain dynamics and persistence. Here we review and evaluate laboratory experiments that tested hypotheses developed from these theoretical studies. This review specifically focuses on the effects of inducible defenses in phytoplankton-rotifer food chain dynamics. First, we describe the occurrence of colony formation within different strains of green algae (Scenedesmaceae) in response to infochemicals released during grazing by the herbivorous rotifer Brachionus calyciflorus. Then we examined the effects of inducible defenses on the population dynamics of this planktonic system
\end{abstract}

Guest editors: S. S. S. Sarma, R. D. Gulati, R. L. Wallace, S. Nandini, H. J. Dumont \& R. Rico-Martínez

Advances in Rotifer Research

I. Van der Stap $(\bowtie) \cdot$ W. M. Mooij

Department of Food Web Studies, Centre for Limnology,

Netherlands Institute of Ecology (NIOO-KNAW),

Rijksstraatweg 6, Nieuwersluis 3631 AC,

The Netherlands

e-mail: i.vanderstap@nioo.knaw.nl

M. Vos

Department of Biology, University of Victoria, P.O. Box 3020, Victoria, BC, CanadaV8W 3N5

Present Address:

M. Vos

Centre for Estuarine and Marine Ecology, Netherlands Institute of Ecology (NIOO-KNAW), Korringaweg

7Yerseke 4401 NT, The Netherlands in which algal strains that differed in their defense strategies were used. Simple food chains were composed of green algae (Scenedesmaceae), herbivorous rotifers (Brachionus calyciflorus) and carnivorous rotifers (Asplanchna brightwellii). In this system B. calyciflorus exhibits an inducible defense against predation by developing long postero-lateral spines. Experimental studies showed that inducible defenses, as opposed to their absence, could prevent highamplitude population fluctuations. We discuss the dual effects of induced defenses on extinction probabilities and consider the fit of a theoretical model to experimental data to understand the mechanisms that underlie the observed dynamics.

Keywords Asplanchna - Brachionus · Food web · Infochemicals - Kairomones - Phenotypic plasticity · Predator-prey interactions - Scenedesmus

\section{Introduction}

Inducible defenses are phenotypic changes in prey organisms in response to cues associated with presence of a predator. Their aim is to reduce loss rates in subsequent attack by these predators. These strategies are used by a variety of aquatic organisms including algae and both vertebrate and invertebrate animals. Inducible defenses include responses in (1) morphology, e.g., increased body depth in crucian carp in response to piscivorous fish, such as pike 
(Brönmark \& Miner, 1992), (2) behavior, e.g., diel vertical migration in daphnids in response to visually hunting zooplanktivorous fish (Ringelberg, 1991) and (3) life history, e.g., a reduced size at first reproduction in daphnids against fish predation (Stibor \& Lüning, 1994).

\section{Linking levels of integration}

Inducible defenses modify the interaction strength between predator and prey and are as such individuallevel responses that affect the functioning of populations and communities (e.g., Vos et al., 2002). Interesting research topics at the individual level include the costs and benefits of inducible defenses and the resulting contribution of these defenses to fitness. Benefits manifest themselves in reduced predation rates if the defense is actually effective at preventing ingestion. In many cases, however, the defense provides only a partial protection against predation (Vos et al., 2004a, b). Moreover, in several cases it has been difficult to document the actual costs of the induced defenses. This raises the question as to why these defenses have not evolved into constitutive defenses. It could be the case that costs manifest themselves more clearly in nature than in the lab.

At the food web and community levels, most attention has been directed at the effects of inducible defenses on stability and persistence. In an unstable system that shows strong population oscillations species might for instance be lost in the troughs of population cycles.

\section{Theoretical predictions}

Vos et al. (2004a, b) made a theoretical study of the effects of inducible defenses on bi- and tritrophic food chains using a model parameterized specifically for algae-rotifer dynamics. These researchers employed values from the literature as well as experimental data and made qualitative predictions about the consequences of inducible defenses for the stability and persistence of these food chains. Presence of inducible defenses as opposed to no inducible defenses or constitutive defenses increased the area of stable coexistence of algae and rotifers over a range of environmental conditions (Vos et al., 2004a) (Fig. 1). Theoretical predictions also showed that inducible defenses increased the relative impor-
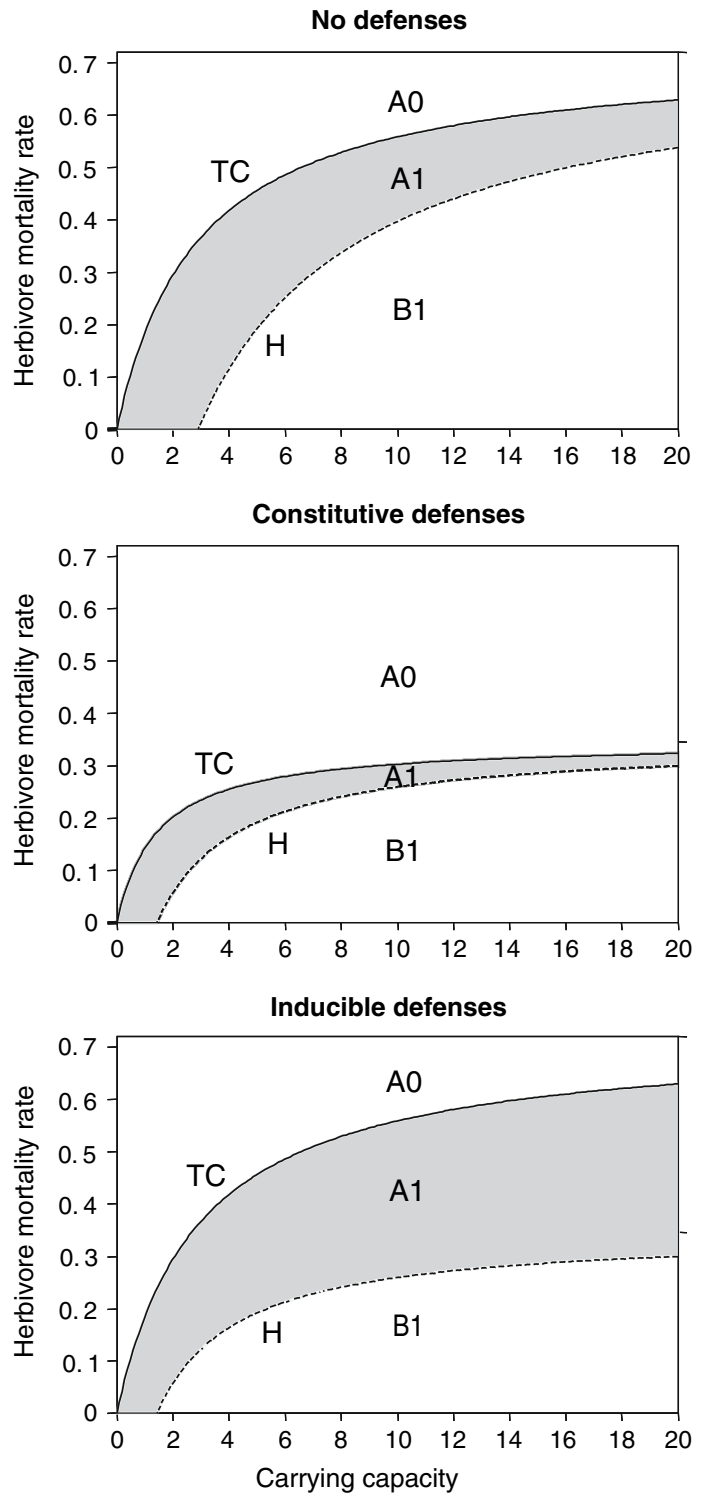

Fig. 1 The stability boundaries of bitrophic food chains without defenses (top), with permanent defenses (middle), or with inducible defenses (bottom), for a range of the carrying capacity and the herbivore mortality rate. The transcritical (TC) and Hopf $(\mathrm{H})$ bifurcation curves separate areas with qualitatively different dynamics. Area A0: only algae exist. Grey area A1: stable coexistence of algae and herbivores. Area B1: algae and herbivores fluctuate. Reproduced, with permission, from Vos et al. (2004a )

tance of bottom-up control. In addition, variation in consumption rates on defended and undefended prey types caused the biomass of all trophic levels to increase simultaneously in response to enrichment (Vos et al., 2004b). 
Here we review both theoretical and experimental studies on inducible defenses and their impact on biand tritrophic food chains that involve algae-rotifer relationships. We describe population level effects of the inducible morphological response of green algae (Scenedesmaceae) against predation by the herbivore Brachionus calyciflorus.

\section{Observed predator-induced defenses in rotifers and algae}

Defenses in herbivorous rotifers

Rotifers are preyed upon by a variety of invertebrate predators including protists, cnidarians, cladocerans, cyclopoid, calanoid and harpacticoid copepods, mysids, insect larvae (species of Chaorboiridae), and other rotifers (Williamson, 1983). Williamson's review of invertebrate predation on rotifers was updated by Brandl (2005) who discussed various factors determining predation rates, as well as the impact of freshwater copepods on rotifers. These and other works demonstrate numerous examples of defenses in rotifers including the following: (1) changes in morphology (e.g., spine development or elongation; reviewed in Gilbert, 1999); (2) behavioral response (e.g., vertical migration or escape reactions; Gilbert \& Kirk, 1988); (3) life history changes (e.g., high maximum growth rate; Walz, 1995); and (4) chemical deterrents (Felix et al., 1995; Walsh et al., 2006). One of the first reports of inducible defenses in rotifers was the induction of spine formation in $B$. calyciflorus by Asplanchna (Gilbert, 1966; Gilbert, 1967). The observations by Gilbert (1967) suggested that the chemical signal (a kairomone) involved in this induction of defenses is a heat-stable protein. More on the chemical ecology of rotifers can be found in Snell (1998).

Juvenile B. calyciflorus and adults with no or short postero-lateral spines are highly susceptible to Asplanchna predation, but those with long spines are relatively protected from being captured or ingested (Gilbert, 1966; Gilbert, 1967; Halbach, 1971). These authors demonstrated that Asplanchna must orient the long-spined prey in such a way as to allow passage of the relatively large prey into its mouth. Time spent swallowing spined prey therefore increases, and ingestion attempts can be unsuccessful.
Young Asplanchna especially have difficulty ingesting spined B. calyciflorus (Gilbert, 1967; Gilbert, 1980). The effects of prey vulnerability on the functional response and growth have been tested for Asplanchna preying on B. calyciflorus and several other rotifer species (Iyer \& Rao, 1996; Nandini et al., 2003). The capture success and ingestion of Asplanchna on spined B. calyciflorus is much lower than on non-spined B. calyciflorus (Iyer \& Rao, 1996). However, a direct comparison of functional responses of Asplanchna on spined and non-spined $B$. calyciflorus has not yet been made. The effectiveness of Brachionus spines in preventing ingestion is likely to differ for different Asplanchna species.

Response to rotifer predation in Scenedesmus

Inducible defenses are common among herbivorous rotifers that often fall prey to carnivorous zooplankton, but even the prey of herbivorous zooplankton are well known to exhibit their own form of inducible defense. For example, species in the Scenedesmaceae, a group of green algae, show striking phenotypic plasticity when in the presence of the microcrustacean Daphnia magna (Hessen \& Van Donk, 1993). The authors observed induction of colony-formation in an algal species that is now called Desmodesmus subspicatus. Subsequently, induction of colony-formation was also found in a Scenedesmus acutus (now S. obliquus) (Lampert et al., 1994). In response to the presence of infochemicals released, due to grazing by this herbivore, the alga formed four- to eight-celled colonies. This response also occurs when Scenedesmus is exposed to grazing-released infochemicals of the rotifers Brachionus and Keratella (Lürling \& Van Donk, 1997; Van Donk et al., 1999). A whole range of different Scenedesmus strains was used in bio-assays to test their response to $B$. calyciflorus (Verschoor et al., 2004a). Most algal strains showed a similar response to infochemicals released by $B$. calyciflorus and D. magna; colony-formation may, therefore be a general response to herbivory in these algae.

Research has shown that induction of colony formation in unicellular algae can have a large effect on feeding efficiency in B. calyciflorus. The maximum ingestion rate of this rotifer was lower when feeding on algae with an induced defense than on 
undefended algae (Verschoor et al., in press). This difference in maximum ingestion rates was, due to a difference in handling time rather than a difference in capture efficiency. Alternatively, algae that exhibited constitutive defenses not only reduced the maximum ingestion rate, but also seemed to be assimilated less efficiently by rotifers. Growth experiments provided further support that larger algae caused reduced Brachionus growth (Mayeli et al., 2004; Lürling et al., 2005).

\section{Observations on bi- and tritrophic food chains with induced defenses}

Overview of population level experiments

Algal-rotifer systems are ideal models to study effects of defenses on the population dynamics of higher trophic levels. Mayeli et al. (2004) presented data on the growth rates of B. calyciflorus and Brachionus patulus using two species of Scenedesmaceae. Here $B$. calyciflorus was able to grow on cells of $S$. obliquus as it did on the green alga Chlorella (Lucía Pavón-Meza et al., 2001). However, it had a lower or even negative population growth coefficient when feeding on the large, colonial and spined $D$. quadricauda. On the other hand, B. calyciflorus grown with the unicellular Chlorella showed different types of population dynamics, including predator-prey cycles, coexistence at equilibrium, or extinction, depending on the conditions of the chemostat set-up (Fussmann et al., 2000). The rotifer-algal cycles in this system seemed to be affected by rapid evolution in response to oscillating predator density (Yoshida et al., 2003). Population dynamics are not only governed by predation, but likely also by initiation of sexual reproduction and diapausing resting eggs in monogonont rotifers (Schröder, 2005). This feature can be adaptive for survival in varying environmental conditions. In laboratory populations rotifers tend to lose the ability to reproduce sexually. The evolution of the rotifer population toward asexual reproduction was a critical component in laboratory studies of algalrotifer dynamics (Fussmann et al., 2003). Recent advances of other mechanisms underlying plankton population dynamics such as complex interactions, and ecological stoichiometry are reviewed in Yoshida (2005).
Bitrophic food chains

Van der Stap et al. (2006) assessed how different algal defenses can lead to qualitatively different patterns in population dynamics. They conducted a bitrophic experiment using food chains composed of four strains of Scenedesmaceae, differing in their defense strategies and an herbivorous species of Brachionus (Fig. 2a). Population fluctuations and herbivore persistence were observed in bitrophic food chains without defenses in algae (Fig. 3a). In the treatment with the constitutively defended algal strain, an increase in population density was followed by extinction of the rotifers in all replicates (see for example Fig. 3d). Such extinctions occurred in only half of the replicates that had algae with induced defenses (as exemplified in Figs. 3b-c). However, the herbivores persisted throughout the experiment in food chains with undefended algae. There were some population fluctuations in this treatment, but without the very low minimum densities that would endanger Brachionus persistence. Statistical analysis of the data showed that herbivore persistence was positively related to the proportion of small highly edible algae present (Van der Stap et al., 2006).

\section{Tritrophic food chains}

Since natural communities are usually constructed of more than two trophic levels, a third trophic level was added to the model system (Verschoor et al., 2004b) (Fig. 2b). The simple food webs were composed of green algae (Scenedesmaceae), herbivorous rotifers (B. calyciflorus) and carnivorous rotifers (Asplanchna brightwellii). In this system $B$. calyciflorus exhibits an inducible defense against predation by developing long posteriolateral spines. At high phosphorus levels, we observed strong population fluctuations in all food chains with undefended algae (Fig. 4a). In one out of three cases this led to the extinction of the top predator. When this treatment was repeated in time, again one out of three replicates ended in extinction of the top-predator after a very low population minimum of its prey. These fluctuations did not occur when algae had inducible defenses (Fig. 4b). At low phosphorus levels, deterministic consumer extinctions were observed, while the algae grew to carrying capacity. Herbivores in food chains with undefended algae showed an oscillatory ten- 

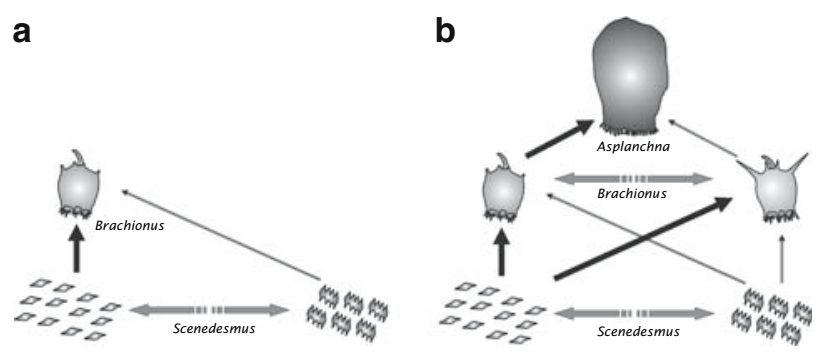

Fig. 2 Composition of bitrophic (a) and tritrophic (b) food chains. Solid arrows show the strength of the interactions. Dashed arrows reflect shifts in morphology between defended and undefended state
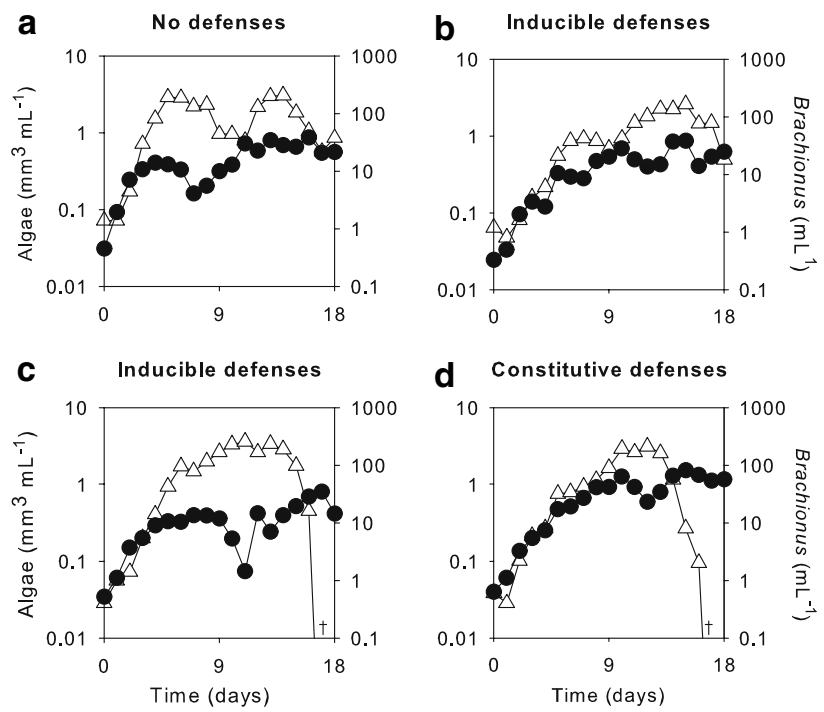

Fig. 3 Population dynamics of bitrophic food chains composed of the herbivorous rotifer Brachionus calyciflorus and the undefended algae Desmodesmus subspicatus (a), inducible defended algae Scenedesmus obliquus II (b), and Scenedesmus obliquus I (c), and constitutively defended algae Desmodesmus

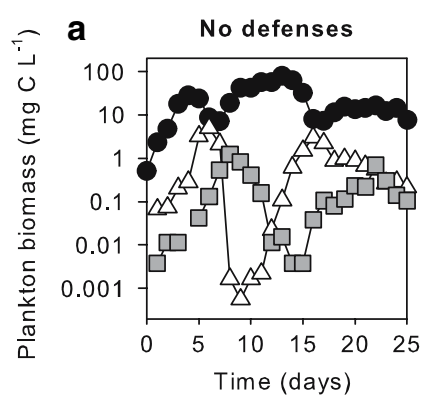

quadricauda (d). The left axis is used for the density of algal volumes (filled circles), the right axis for the density of herbivores (open triangles); one replicate shown. Herbivore extinctions are marked with $\dagger$. Reproduced, with permission, after Van der Stap et al. (2006)

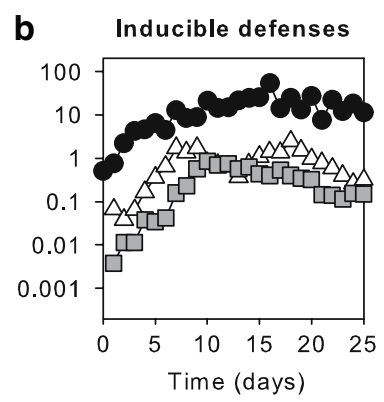

Fig. 4 Population dynamics of tritrophic food chains in the high phosphorus treatments, with densities expressed as $\mathrm{mg} \mathrm{C} \mathrm{l}^{-1}$. Circles denote phytoplankton, triangles herbivorous zooplankton (Brachionus) and squares carnivorous zooplank-

ton (Asplanchna). (a) Food chain with undefended phytoplankton (Desmodesmus); (b) food chain with inducible defenses in phytoplankton (Scenedesmus); one replicate shown. Reproduced, with permission, after Verschoor et al. (2004b) 
dency before going extinct. In the low phosphorus treatment with inducible defenses, herbivores went extinct in a more monotonical way (Verschoor et al., 2004b).

\section{Chemostat}

Predator-prey dynamics stabilized in a one-reactor chemostat with a mixed culture of $B$. calyciflorus and S. obliquus. This coincided with the induction of colony-formation in the algal prey. In contrast, highamplitude population fluctuations were observed in the second reactor of a two-stage chemostat where prey were unable to form protective colonies (Lürling et al., 2005). Thus, we see that the use of continuous culture systems, especially for rotifers, is highly useful for the study of general population regulation principles (Walz, 1993).

\section{Linking experimental observations and the theoretical work}

The model by Vos et al. (2004a) predicted stability of predator-prey interactions for only a narrow range of parameter values when food chains were based on undefended algae. In contrast, for food chains with inducible defenses, stable coexistence of predator and prey was predicted for a relatively wide range of conditions. The experimental observations of Verschoor et al. (2004b) can be interpreted in this context. Here we note that the actual dynamics were high-amplitude fluctuations, which seemed to dampen out in the case of undefended algae at the base of the food chain, while a monotonic approach to a stable equilibrium was seen in the case of algae protected by inducible defenses (Fig. 4). The latter agrees directly with our expectation as based on the model: inducible defenses seem to stabilize dynamics. However, the dynamics in food chains with undefended algae can be explained in two alternative ways. The first possibility is that dynamics were truly cyclic, but some factor (such as increased heterogeneity through accumulation of detritus) stabilized the system late in the experiment. This explanation is consistent with our observations. However, it is also possible that the dynamics were inherently stable, but approached equilibrium through a damped oscillation, instead of monotonically. In this case, the dynamics of systems with undefended versus algae protected by inducible defenses are not separated by a Hopf bifurcation, but by a stable focus-node boundary, which is situated within the stability area itself (Yodzis \& Innes, 1992; Vos et al., 2005). On one side of this line a stable equilibrium is approached monotonically, on the other side this occurs through a damped oscillation (Vos et al., 2005). We cannot formally exclude this possibility based on current evidence, but further chemostat experiments will be helpful in teasing these alternative explanations apart. The experimental results of Verschoor et al. (2004b) clearly showed that inducible defenses in algae prevented high-amplitude population fluctuations in both bitrophic and tritrophic food chains, but the exact nature of these fluctuations remains unclear. The experiments showed that the algae-rotifer model system is very reproducible within batch, semicontinuous and continuous cultures, but dynamics may differ between these experimental systems; therefore, caution is warranted when making extrapolations to nature based on any of these systems alone. A particularly interesting observation is that inducible defenses at the herbivore level had little or no effect on stability in the model of Vos et al. (2004a; b). Exactly the same surprising result was obtained in the experiments by Verschoor et al. (2004b). These results await an ecological explanation.

In order to identify, whether phenotypic plasticity at the individual level has consequences for community dynamics, a more quantitative approach was taken and mechanistic predator-prey models were fitted to experimental population dynamics data collected in batch cultures by Van der Stap et al. (unpublished observations). They started off by using the inducible defense model by Vos et al. (2004a; b), however, realizing that this model assumes constant conditions, it was modified for application to the transient dynamics in a batch experiment. A good match was obtained between model and data, with $77 \%$ of the variance in the data being accounted for by the model. Moreover, results from the model showed that quantitative differences in handling times of prey placed the system at different locations in parameter space, in particular relative to the predator existence boundary. Long handling times on defended prey brought predators closer to the existence boundary, where they can go extinct earlier when nutrients are being depleted in the system than 
predators that are further away from the existence boundary.

\section{Other predator-prey interactions}

Where predators and prey live together, predator responses to prey defenses may be expected. Asplanchna priodonta is able to grow larger jaws (Fontaneto \& Melone, 2005) and this may be one way of how a predator can respond to spines in brachionid prey. A response in jaw size has not yet been studied or observed for the experimental predator species $A$. brightwellii. Besides the inducible defense of spines in B. calyciflorus, two other species in this genus employ predatory defense strategies. Brachionus rubens does not exhibit these morphological defenses, rather it has the ability to attach to cladocerans, which may serve, as an alternative escape mechanism from predation by Asplanchna (Iyer \& Rao, 1996). Rotifers of the Brachionus plicatilis complex differ in body size and vulnerability to copepod predation. This differential sensitivity to predation promoted coexistence in a situation, where the competitively dominant species were preferred as prey (Ciros-Pérez et al., 2004).

\section{Conclusion}

Phenotypic plasticity in individual prey can have profound effects on population dynamics, affecting both consumers at higher trophic levels and the prey themselves. Plasticity in defenses creates variation in handling times and/or attack rates of predators on different prey types. This can cause prey with inducible defenses, as opposed to those without defenses, to prevent strong population fluctuations in both bi- and trirophic food chains. However, these differences in prey also place model predators at distinct locations in parameter space, which in turn confer different risks of extinction. Whereas induced defenses clearly promote stability and persistence under high nutrient conditions, this is no longer the case when these nutrients become depleted and the environment becomes harsher for predators. In such an environment, predators may actually go extinct sooner, when basal preys in the food chain are more strongly defended.
Acknowledgements We would like to thank Robert L. Wallace and two anonymous reviewers for their helpful comments on the manuscript. I.v.d.S. was supported by Earth and Life Sciences grant 812.04.008 from the Netherlands Organisation for Scientific Research (NWO-ALW). Publication 4111 Netherlands Institute of Ecology (NIOO-KNAW).

\section{References}

Brandl, Z., 2005. Freshwater copepods and rotifers: predators and their prey. Hydrobiologia 546: 475-489.

Brönmark, C. \& J. G. Miner, 1992. Predator-induced phenotypical change in body morphology in crucian carp. Science 258: 1348-1350.

Ciros-Pérez, J., M. J. Carmona, S. Lapesa \& M. Serra, 2004. Predation as a factor mediating resource competition among rotifer sibling species. Limnology and Oceanography 49: 40-50.

Felix, A., M. E. Stevens \& R. L. Wallace, 1995. Unpalatability of a colonial rotifer, Sinantherina socialis to small zooplanktivorous fishes. Invertebrate Biology 114: 139-144.

Fontaneto, D. \& G. Melone, 2005. Do rotifer jaws grow after hatching? Hydrobiologia 546: 213-221.

Fussmann, G. F., S. P. Ellner \& N. G. Hairston, 2003. Evolution as a critical component of plankton dynamics. Proceedings of the Royal Society of London Series BBiological Sciences 270: 1015-1022.

Fussmann, G. F., S. P. Ellner K. W. Shertzer \& N. G. Hairston, 2000. Crossing the Hopf bifurcation in a live predatorprey system. Science 290: 1358-1360.

Gilbert, J. J., 1966. Rotifer ecology and embryological induction. Science 151: 1234-1237.

Gilbert, J. J., 1967. Asplanchna and postero-lateral spine production in Brachionus calyciflorus. Archiv Fur Hydrobiologie 64: 1-62.

Gilbert, J. J., 1980. Feeding in the rotifer Asplanchna: behavior, cannibalism, selectivity, prey defenses and impact on rotifer communities. In Kerfoot, W. C. (ed.), Evolution and Ecology of Zooplankton Communities. University Press of New England: 158-172.

Gilbert, J. J., 1999. Kairomone-induced morphological defenses in rotifers. In Tollrian, R. \& C. D. Harvell (ed.), The Ecology and Evolution of Inducible Defenses. Princeton University Press, Princeton New Jersey: 127141.

Gilbert, J. J. \& K. L. Kirk, 1988. Escape response of the rotifer Keratella: description, stimulation, fluid-dynamics, and ecological significance. Limnology and Oceanography 33: 1440-1450.

Halbach, U., 1971. Zum Adaptivwert der zyklomorphen Dornenbildung von Brachionus calyciflorus Pallas (Rotatoria). Oecologia 6: 267-288.

Hessen, D. O. \& E. Van Donk, 1993. Morphological changes in Scenedesmus induced by substances released from Daphnia. Archiv für Hydrobiologie 127: 129-140.

Iyer, N. \& T. R. Rao, 1996. Responses of the predatory rotifer Asplanchna intermedia to prey species differing in vulnerability: laboratory and field studies. Freshwater Biology 36: 521-533. 
Lampert, W., K. O. Rothhaupt \& E. von Elert, 1994. Chemical induction of colony formation in a green alga (Scenedesmus acutus) by grazers (Daphnia). Limnology and Oceanography 39: 1543-1550.

Lucía Pavón-Meza, E., S. S. S. Sarma \& S. Nandini, 2001. Effect of different densities of live and dead Chlorella vulgaris on the population growth of rotifers Brachionus calyciflorus and Brachionus patulus (Rotifera). Revista de Biologia Tropical 49: 895-902.

Lürling, M., H. Arends, W. Beekman, M. Vos, I. Van der Stap, W. M. Mooij \& M. Scheffer, 2005. Effect of grazer-induced morphological changes in the green alga Scenedesmus obliquus on growth of the rotifer Brachionus calyciflorus. Proceedings of the international association of theoretical and applied limnology 29: 698-703.

Lürling, M. \& E. Van Donk, 1997. Morphological changes in Scenedesmus induced by infochemicals released in situ from zooplankton grazers. Limnology and Oceanography 42: 783-788.

Mayeli, S. M., S. Nandini \& S. S. S. Sarma, 2004. The efficacy of Scenedesmus morphology as a defense mechanism against grazing by selected species of rotifers and cladocerans. Aquatic Ecology 38: 515-524.

Nandini, S., R. Perez-Chavez \& S. S. S. Sarma, 2003. The effect of prey morphology on the feeding behaviour and population growth of the predatory rotifer Asplanchna sieboldi: a case study using five species of Brachionus (Rotifera). Freshwater Biology 48: 2131-2140.

Ringelberg, J., 1991. Enhancement of the phototactic reaction in Daphnia hyalina by a chemical mediated by juvenile perch (Perca fluviatilis). Journal of Plankton Research 13: 17-25.

Schröder, T., 2005. Diapause in monogonont rotifers. Hydrobiologia 546: 291-306.

Snell, T. W., 1998. Chemical ecology of rotifers. Hydrobiologia 388: 267-276.

Stibor, H. \& J. Lüning, 1994. Predator-induced phenotypic variation in the pattern of growth and reproduction in Daphnia hyalina (Crustacea, Cladocera). Functional Ecology 8: 97-101.

Van der Stap, I., M. Vos \& W. M. Mooij, 2006. Linking herbivore-induced defences to population dynamics. Freshwater Biology 51: 424-434.

Van Donk E., M. Lürling \& W. Lampert, 1999. Consumerinduced changes in phytoplankton: inducibility, costs, benefits and the impact on grazers. In Tollrian, R. \& C. D. Harvell (ed.), The Ecology and Evolution of Inducible Defenses. Princeton University Press, Princeton New Jersey, 89-103.
Verschoor, A. M., I. Van der Stap, N. R. Helmsing, M. Lürling \& E. Van Donk, 2004a. Inducible colony formation within the Scenedesmaceae: adaptive responses to infochemicals from two different herbivore taxa. Journal of Phycology 40: 808-814.

Verschoor, A. M., M. Vos \& I. Van der Stap, 2004b. Inducible defences prevent strong population fluctuations in bi- and tritrophic food chains. Ecology Letters 7: 1143-1148.

Verschoor, A.M., Y.S. Zadereev \& W.M. Mooij, in press. Infochemical-mediated trophic interactions between the rotifer Brachionus calyciflorus and its food algae. Limnology and Oceanography.

Vos, M., B. J. G. Flik, J. Vijverberg, J. Ringelberg \& W. M. Mooij, 2002. From inducible defences to population dynamics: modelling refuge use and life history changes in Daphnia. Oikos 99: 386-396.

Vos, M., B. W. Kooi, D. L. DeAngelis \& W. M. Mooij, 2004a. Inducible defences and the paradox of enrichment. Oikos 105: 471-480.

Vos, M., B. W. Kooi, D. L. DeAngelis \& W. M. Mooij, 2005. Inducible defenses in food webs. In De Ruiter, P. C., V. Wolters \& J. C. Moore (ed.), Dynamic Food Webs: Multispecies Assemblages, Ecosystem Development, and Environmental Change. Academic Press, Amsterdam: 114-127.

Vos, M., A. M. Verschoor, B. W. Kooi, F. L. Wäckers, D. L. DeAngelis \& W. M. Mooij, 2004b. Inducible defenses and trophic structure. Ecology 85: 2783-2794.

Walsh, E. J., M. Salazar, J. Remirez, O. Moldes \& R. L. Wallace, 2006. Predation invertebrate predators on the colonial rotifer, Sinantherina socialis. Invertebrate Biology 125 : 325-335.

Walz, N. (ed.), 1993. Plankton regulation dynamics: experiments and models in rotifer continuous cultures. Ecological studies. Springer-Verlag, 308 pp.

Walz, N., 1995. Rotifer populations in plankton communities: energetics and life history strategies. Experientia 51: 437453.

Williamson, C. E., 1983. Invertebrate predation on planktonic rotifers. Hydrobiologia 104: 385-396.

Yodzis, P. \& S. Innes, 1992. Body size and consumer-resource dynamics. American Naturalist 139: 1151-1175.

Yoshida, T., 2005. Toward the understanding of complex population dynamics: planktonic community as a model system. Ecological Research 20: 511-518.

Yoshida, T., L. E. Jones, S. P. Ellner, G. F. Fussmann \& N. G. Hairston, 2003. Rapid evolution drives ecological dynamics in a predator-prey system. Nature 424: 303306. 